\title{
FUNCIÓN Y PERFIL PROFESIONAL DE LOS PSICOPEDAGOGOS. UNA PERSPECTIVA DESDE CATALUÑA ${ }^{1}$
}

\author{
Psychology and Pedagogy teachers: their role framework \\ and professional profile. A Catalan view
}

\section{Enric ROCA CASAS}

Universitat Autònoma de Barcelona. Facultat de Ciències de l'Educació. Departament de Pedagogia Sistemãtica i Social. Edifici G6. 08193. Bellaterra (Barcelona). Institut d'Educació Secundària Antoni Pous i Argila. Avda. Roma, 260. 08560 Manlleu.Barcelona.Correo-e: enric.roca@uab.es

Fecha de aceptación definitiva: marzo de 2001

BIBLID [(1130-3743) 12, 2000, 159-178]

\section{RESUMEN}

En Cataluña, al igual que en otras comunidades autónomas, las funciones de los nuevos profesores de Psicología y Pedagogía de la Educación Secundaria se han ido regulando desde 1995 por medio de las denominadas "normativas de curso" que año tras año ha ido publicando el departamento de Educación del Gobierno autónomo.

Se detecta un proceso de disminución de las funciones de asesoramiento y coordinación y un aumento de la dedicación a la docencia de grupos de alumnos con retraso escolar de las materias instrumentales. Eso supone, a nuestro entender, no optimizar las posibilidades profesionales de los psicopedagogos en los institutos de Secundaria. Sin embargo, gracias a la previsión de los denominados "planes estratégicos de

1. El contenido de este artículo está basado en la comunicación: «Evaluación crítica de la política educativa reguladora de las funciones y el perfil profesional de los psicopedagogos en los IES de Cataluña" presentada por el autor en el vill Congreso Nacional de Teoría de la Educación: Evaluación de políticas educativas celebrado en Huelva los días 21, 22 y 23 de marzo de 2001. 
centro" que quiere impulsar la Administración catalana, vemos ahora una oportunidad para, a partir de la iniciativa de los propios centros, diseñar específicamente el perfil profesional de su psicopedagogo y atribuirle aquellas funciones que puedan revertir en la mejora de la calidad del centro, superando ciertos prejuicios y resistencias corporativas de algunos sectores del profesorado y determinados equipos directivos con relación al trabajo pedagógico específico que estos nuevos profesionales, por su formación, deberían ejercer en los centros.

Descriptores: asesoramiento psicopedagógico, atención a la diversidad, Departamento de Orientación, Educación Secundaria Obligatoria, evaluación psicopedagógica, orientación educativa.

\section{SUMMARY}

Since 1995 onwards, in Catalonia and in other "comunidades autónomas", the responsibilities of the Secondary School new teachers of Psychology and Pedagogy, have become ruled by the called "course regulations". This rules has been published by the Catalan Government Educational Department.

In the content of such responsibilities, there are first of all a decrease of the assessment and co-ordination aspects. Secondly, the responsibilities of the teachers are increasingly focused in the learning process of children groups with difficulties in instrumental subjects. Due to this, we believe that this evolution implies a low capabilities optimisation of such teachers in the Secondary School. However, "the strategic centre plans" that are being designed by the Catalan Administration could improve this low capabilities optimisation of such teachers. Through this plan each centre will be able to design its teacher of Psychology and Pedagogy requirements and responsibilities to improve its school quality. At the same time, prejudices of some managerial teams and those of some teachers involved in other educational areas will be overcome. The consequence is that the teachers in Psychology and Pedagogy will achieve their right place in schools.

Key words: psychological and pedagogical advice, attention to the diversity, Guidance Department, Secondary School Education, education evaluation, education guidance.

\section{INTRODUCCIÓN}

En 1993 nacía la nueva licenciatura de Psicopedagogía y un año más tarde se producían las primeras oposiciones para ocupar plazas de profesores de Psicología y Pedagogía en los institutos de Educación Secundaria (IES). Desde entonces el camino que en Cataluña, y suponemos que en otras comunidades autónomas y en el mencionado territorio MEC, han recorrido estos nuevos profesionales en los institutos se parece al que tuvieron que emprender aquellos exploradores europeos que en los siglos Xv y XVI descubrían nuevas sorpresas, no siempre agradables, en 
cada expedición y en cada inesperado territorio que encontraban o, mejor dicho, que se les aparecía por sorpresa, pues, casi siempre era otro distinto al que buscaban. Los avatares, retos, equilibrios, sacudidas, decepciones y algunas desesperaciones y muchos desconciertos han acompañado a estos nuevos profesionales por sus primeras andaduras como profesores con perfil propio en la, también, nueva etapa de la Educación Secundaria Obligatoria.

Para bien y para mal su camino se ha entrelazado tanto con la reforma educativa que muchos de los problemas, de las angustias y de las decepciones que arrastran se pueden achacar a las incertidumbres, incoherencias, titubeos y heridas que la aplicación de la reforma, sobre todo en los institutos de Secundaria que antes lo eran de Bachillerato, ha dejado al descubierto reflejando de forma explícita y clara el débil calado pedagógico real que, en muchos de los antiguos profesores de Secundaria pero también de los nuevos, ha producido la LOGSE y sus implicaciones dirigidas a crear una nueva cultura de la profesión docente.

\section{MARCO NORMATIVO}

En la $L O D E$ (1985) se reconoce como un derecho básico de todo alumno el recibir una adecuada orientación escolar y profesional (Título preliminar, artículo 6.1., apartado $f$ ). Por su parte la LOGSE (1990) establece una serie de principios por los que se debe regir la actividad educativa. Entre ellos se cita explícitamente la atención psicopedagógica y la orientación educativa y profesional (Título preliminar, artículo 2.3., apartado g). Vemos, pues, que se ha añadido la "atención psicopedagógica" como un principio que ha de garantizar la actividad educativa. La LOGSE no menciona a ningún profesional u órgano específico que se deba hacer cargo de la orientación escolar y profesional ni de la atención psicopedagógica. Pero la propia conceptualización de este último término está indicando implícitamente que los profesionales en Psicopedagogía podrían ser los más idóneos para realizar esta tarea. En el capitulo $v$ de la ley, el referido a la educación especial, se dice que el sistema educativo ha de disponer de los recursos necesarios para que los alumnos con necesidades educativas especiales puedan alcanzar los mismos objetivos establecidos para todos y hacerlo dentro del mismo sistema (artículo 36.1.). Más adelante se señala que para alcanzar estos fines el sistema educativo deberá proveerse "de profesores de las especialidades correspondientes y de profesionales cualificados" (artículo 37.1.). En el título IV de la ley, y en referencia a la calidad de la enseñanza, se señala como uno de los factores que la favorecen: "la orientación educativa y profesional. (artículo 55., punto e). Más tarde se afirma: da tutoría y orientación de los alumnos formará parte de la función docente. Corresponde a los centros educativos la coordinación de estas actividades. Cada grupo de alumnos tendrá un profesor tutor (artículo 60.1.) Todo parece indicar que se está señalando indirectamente al profesor tutor como el principal responsable de la función orientadora. En el siguiente punto se dice que "las administraciones educativas 
garantizarán la orientación académica, psicopedagógica y profesional de los alumnos (...) La coordinación de las actividades de orientación se llevará a cabo por profesionales con la debida preparación" (artículo 60.2.). Aquí aparece la mención "psicopedagógica" referida a la orientación y no a la atención, valoración o evaluación y se habla de profesionales con una preparación determinada que puede interpretarse como diferenciada de la de los tutores. Pero aquello que, precisamente, los distingue es la aparición del término "orientación psicopedagógica". Es decir, la ley resulta ambigua con relación al tipo de profesional sobre el que debe recaer la responsabilidad principal de la orientación educativa y profesional. Además, ¿la orientación "educativa" se entiende aquí como la suma de la orientación "personal" más la orientación "académica? ¿O más bien como sinónimo de esta última? Resulta significativo que cuando aparece "psicopedagógico/a" a continuación se hable de "profesionales con la debida preparación". Con todo ello, parecería que se estaba preparando abrir las puertas de la Educación Secundaria a los psicopedagogos $^{2}$, pero sin mencionarlo directamente, ni tampoco dejando claros y delimitados los posibles ámbitos de intervención en un centro donde tutores. y diferentes órganos de gobierno, dispondrán también de atribuciones y responsal)ilidades relacionadas con las tareas de orientación y atención a la diversidad del alumnado.

Esta primera indefinición de la ley ha supuesto posteriormente, al menos en Cataluña, toda una tradición de normas ambiguas que aún condicionan diariamente el trabajo de los psicopedagogos en los centros de Secundaria. A partir de aquel marco legal general, cada comunidad autónoma con competencias en educación instauró los decretos que desarrollaban tanto la LOGSE como el margen de autonomía curricular que les correspondía respetando las enseñanzas mínimas prescritas por el Gobierno central. Posteriormente se aprobó la tercera ley orgánica sobre educación que rige actualmente nuestro sistema educativo: la LOPEGCE (1995). Una de sus principales finalidades era desarrollar el título iv de la LOGSE ya mencionado anteriormente. Por ello, en la exposición de motivos de la Ley de 1995 se recuerdan los factores que favorecen la calidad de los centros y la obligación de los poderes públicos de prestar hacia ellos su actuación prioritaria. Se vuelven a mencionar los mismos del artículo 55 de la LOGSE, entre ellos, la orientación educativa y profesional, pero la ley sólo desarrollará los apartados de la función directiva (apartado c), la inspección educativa (apartado f), la evaluación del sistema educativo (apartado g), además del de la mejora de la participación educativa y el gobierno de los centros. Por lo tanto, no se desarrollan los factores de la formación del profesorado (apartado a), la programación docente (apartado b), la innovación

2. A partir de ahora utilizaremos el término psicopedagogo para referirnos concretamente a los profesores de la especialidad de Psicología y Pedagogía de la Educación Secundaria a menos que indiquemos lo contrario.

(C) Ediciones Universidad de Salamanca

Teor. educ. 12, 2000, pp. 159-178 
y la investigación educativa (apartado d) y, en ningún caso, la orientación educativa y profesional (apartado e) que figuraban a la LOGSE.

En el caso de Cataluña, será en el Decreto 199/1996, de 12 de junio, donde el Gobierno autónomo actualizará y fijará un reglamento orgánico de los centros docentes públicos que imparten la Educación Secundaria y la Formación Profesional de grado superior, desarrollando y ampliando, dentro de sus competencias, el redactado de la LOPEGCE. En este reglamento, entre otras muchas cuestiones, se fijan las funciones del profesor tutor en los institutos de Educación Secundaria. Entre ellas figura la de llevar a cabo las actividades "de información y de orientación académica de los alumnos" (artículo 52.2., apartado e). No se habla, ni aquí ni en todo el decreto, de la atención (orientación, evaluación...) psicopedagógica como tampoco se hace ningún tipo de referencia a la labor o a la mera existencia de los psicopedagogos en los institutos. El decreto hace responsable al jefe de estudios del centro de coordinar la programación de la acción de los tutores y hacer su seguimiento (artículo 16.2., apartado e). Por lo tanto, se supone que será responsable también de la orientación académica - y suponemos que profesional- que, como hemos visto, es una de las funciones propias del tutor. Por cierto, la que antes era orientación "educativa" ahora es "académica" (¿la académica incluye la orientación "personal" o se trata solo de la orientación "escolar"?). En el caso, pues, que los psicopedagogos realizaran alguna función específica con relación a la orientación académica de los alumnos ¿deberían coordinarse éstos con el jefe de estudios? En Cataluña aquellos que pueden «ayudar a los centros a diseñar y aplicar las actividades de tutoría y de orientación, en el marco del Plan de Acción Tutorial»3 (PAT), así como también ofertar actividades de orientación e información al alumnado, son los Equipos de Asesoramiento Psicopedagógico (EAP). Estos equipos representan servicios de asesoramiento externos a los centros del Departamento de Enseñanza del Gobierno de Cataluña. Pero estos equipos, según las mismas instrucciones -y también las de los años siguientes-, han de desarrollar sus funciones en los centros de Secundaria en "estrecha colaboración" ${ }^{4}$ con el psicopedagogo del centro. Por eso cabría suponer que este profesional tendría algo que decir en los temas de orientación y acción tutorial de los institutos de Secundaria.

Otro órgano unipersonal de gobierno de los institutos es el coordinador pedagógico, que tiene, entre sus funciones, la de velar por la elaboración de las adecuaciones y modificaciones curriculares necesarias para atender a los alumnos con necesidades educativas especiales y, en general, para atender la diversidad de los ritmos de aprendizaje y la singularidad de cada alumno" (artículo 20.2., apartado, d). Resulta evidente que el psicopedagogo del centro dependerá fundamentalmente

3. Instrucciones de la Dirección General de Ordenación Educativa para la organización y el funcionamiento de los servicios educativos del Departamento de Enseñanza para el curso 1997-98. Full de disposicions $i$ actes administratius, p. 1.214

4. Ibídem, p. 1.214. 
del coordinador pedagógico que es quien tiene la responsabilidad de efectuar el seguimiento y la evaluación de todas las acciones educativas que se realizan en el instituto.

Éste es, de forma resumida, el marco normativo de los centros de Secundaria de Cataluña donde se incorporan, a partir de 1994, los psicopedagogos como nuevos profesores de la educación Secundaria.

3. EVOluCión DE LAS DIVERSAS "NORMATIVAS DE CURSO" REGULADORAS DE LAS FUNCIONES DE LOS PSICOPEDAGOGOS EN LOS IES

Analizaremos a continuación cómo la Administración educativa catalana ha ido regulando las funciones de los psicopedagogos en los centros de Secundaria durante los últimos cursos y cómo, a partir de la vacilante y ambigua adecuación con el marco que expusimos en el apartado anterior, su perfil, posibilidades y limitaciones profesionales han ido variando con relación a las propias oscilaciones administrativas removidas según soplaran más o menos fuertes los vientos que impulsaban multitud de influencias de diversos orígenes e intereses.

Las funciones de los psicopedagogos que la Administración catalana ha ido definiendo a partir de las diversas normativas de curso y que han permanecido más o menos invariables desde 1995 hasta el curso actual serían, de forma resumida, las siguientes:

a) Atención a alumnos con más dificultades para aprender impartiendo docencia en pequeño grupo o, excepcionalmente, de forma individual. También comporta esta atención la preparación de materiales y actividades adaptadas en colaboración con el resto del profesorado.

b) Impartir docencia al alumnado con más dificultades para aprender en la parte común ${ }^{5}$ del currículum (grupos reducidos, desdoblamientos y otras formas de organización en, principalmente, las materias de tipo instrumental).

c) Impartir docencia en la parte variable del currículum en créditos relacionados con los aspectos de la Psicología y la Pedagogía (conocimiento de la persona, interacción e inserción en el medio social, transición al mundo laboral, desarrollo de habilidades cognitivas, etc.).

d) Responsable de coordinar las actividades docentes que el centro establezca para la atención específica del alumnado con necesidades educativas especiales.

e) Colaborar con los diversos órganos que trabajan en la organización general de las medidas de atención a la diversidad de los alumnos con necesidades

5. En Cataluña la organización del currículum en Secundaria se divide entre créditos comunes para todo el alumnado (de las materias comunes de la etapa) y créditos variables, unos de ellos tipificados desde la Administración y otros de libre oferta de los centros. Todos ellos tienen una duración trimestral. 
educativas de acuerdo con las previsiones establecidas en el PCC (Proyecto Curricular de Centro).

Este orden en el establecimiento regulador de las funciones de los psicopedagogos en los centros de Secundaria en Cataluña se modificó a partir de las instrucciones del curso 1999-2000 6 , donde en primer lugar aparecían las funciones " $b$ " $\mathrm{y}$ "c" mientras que la " $a$ " pasaba al tercer lugar. Esto no tendría una mayor significación sino fuera porque indica una tendencia determinada distinta a partir de ese curso (1999-2000) en relación al resto de diferencias producidas entre los cursos 19951996 y 1998-1999. Esta tendencia se puede resumir en:

1. Dar prioridad a la docencia con alumnos por delante de las otras funciones. Docencia con relación, sobre todo, a créditos comunes de Lengua (catalana y castellana) y Matemáticas. Esto se relaciona con la mención explícita en la normativa del curso 1999-2000 de considerar al psicopedagogo como "un profesor más del centro y, aun teniendo en cuenta la especificidad de algunas de sus funciones, tiene una dedicación a la atención de alumnos y a las tareas de coordinación análoga al resto de profesores" ?.

2. En la docencia de los créditos variables, antes de mencionar aquellos que tratan de los aspectos de la Psicología y la Pedagogía (ver apartado "C" de las funciones anteriormente relacionadas), se concreta el impartir la docencia en créditos de refuerzo de las materias instrumentales, lo que indica que el psicopedagogo es percibido, en buena parte, como un profesor de refuerzo o de apoyo educativo dirigido a todos los alumnos que no lleguen al nivel de los objetivos de los créditos comunes y, en cambio, no es visto como aquel profesional que debería atender preferentemente a los alumnos con más necesidades para aprender o con necesidades educativas especiales. Hay una gran diferencia de concepto: una cosa es atender a los alumnos con más dificultades (siempre serán una minoría en un centro, lo que explica que haya sólo un psicopedagogo por centro además de poder compartir el trabajo en algunos centros con un maestro de Pedagogía Terapéutica ${ }^{8}$ ) y otra cosa bien distinta es impartir docencia con todos los alumnos que no han obtenido los objetivos básicos de los créditos

6. Para el actual curso 2000-2001 el Departamento de Enseñanza, con relación a las funciones de los psicopedagogos, no ha introducido ninguna mención específica, lo que significa que las funciones son las mismas que el anterior curso 1999-2000.

7. La cursiva es nuestra. Normativa del curso 1999-2000: Resolución de 21 de junio de 1999. Full de Disposicions i Actes Administratius del Departament d'Ensenyament. Nüm. 775, any xvI, juny 1999.

8. En muchos institutos, aprovechando el hecho de poderse incorporar al primer ciclo de Secundaria maestros que provenían de la antigua EGB, sobre todo aquellos que habían ejercido docencia en el ciclo superior, muchos profesores de Pedagogía Tetapéutica que ejercían como maestros de Educación Especial han pasado a los institutos y actúan con una parecida línea de actuación de los psicopedagogos. 
comunes de las áreas instrumentales, donde serían tanto o más validos los mismos profesores del departamento afectado (Lenguaje o Matemáticas).

3. En tercer lugar se menciona la función de la atención individualizada en pequeño grupo a los alumnos con necesidades educativas especiales. Es decir, ha pasado implícitamente de la primera prioridad en todas las normativas precedentes a la tercera a partir del curso 1999-2000. Parece que ahora resulta más prioritaria la docencia en desdoblamientos u otras formas de organización no tan específicas con el pequeño grupo que permita una atención más personalizada.

4. Se insiste que el psicopedagogo es el responsable (!) de coordinar las actividades docentes de atención al alumnado con necesidades educativas especiales pero no se dice cómo lo debe hacer. Para coordinar actividades se supone que uno ha de poder fijar criterios para después supervisar su aplicación y su coordinación. Pero los psicopedagogos en los institutos no tienen ningún poder de supervisión o de fijación de pautas para el conjunto del profesorado. ¿De qué se trata pues? ¿Que el psicopedagogo coordine lo que otros fijan, aplican y supervisan? No queda claro y aquí la normativa diríamos que es expresamente ambigua.

5. También se insiste en que el psicopedagogo ha de "colaborar con los diversos órganos que trabajan en la atención a la diversidad de los alumnos con necesidades educativas. ¿Colaborar aquí qué significa? ¿Asistir a las reuniones de la comisión de atención a la diversidad ${ }^{9}$ como "uno más"? ¿O como psicopedagogo debería tener algún papel relevante en esta comisión? Otra vez una imprecisión calculada en la delimitación de las funciones dentro de la normativa.

Dejando a un lado la clara tendencia de fijar cada vez más las funciones de los psicopedagogos en los institutos como profesionales del refuerzo educativo y como uno más de los profesores que ejercen docencia en el centro con prioridad, eso sí, de hacerlo en los grupos de alumnos con más dificultades (¿dificultades de los alumnos para aprender o dificultades de los profesores para enseñarlos?), otro aspecto que ha contribuido a dar una imagen de improvisación y de desconcierto ha sido el auténtico baile de números que las distintas normativas han ido estableciendo con relación al horario de los psicopedagogos en los institutos.

9. Se "recomienda" que, para planificar y hacer el seguimiento del conjunto de actuaciones y medidas que el centro ha de tomar para atender la diversidad de necesidades educativas de los alumnos, la persona encargada de la coordinación pedagógica (normalmente será el coordinador pedagógico del instituto) establezca una comisión de atención a la diversidad. Esta comisión estará integrada por el psicopedagogo o el maestro de Pedagogía Terapéutica, los jefes de departamento de las áreas instrumentales y la persona del EAP (equipo de asesoramiento psicopedagógico externo) que interviene en el centro (normativa del curso 1998-1999). 
En la normativa del curso 1997-1998 se decía que el psicopedagogo dispondría de una reducción de tres horas semanales de su horario lectivo para dedicarlo a las tareas de coordinación y de colaboración descritas en esa misma normativa, tareas que serían las siguientes:

a) Coordinar las actividadés docentes en referencia a la atención del alumnado con necesidades educativas especiales y coordinar también la tutoría en aspectos tales como las formas de agrupamiento de los alumnos en tareas de refuerzo o de adaptación curricular, en los criterios de orientación de los créditos variables del centro, los procedimientos para evaluar los resultados, etc.

b) Colaborar con los departamentos con relación a las adaptaciones curriculares, con el EAP en la aplicación de su plan de trabajo en el centro, etc.

En la siguiente normativa, la del curso 1998-1999, las funciones eran las mismas pero se establecía que para poder ejercer sus tareas de coordinación y de colaboración ahora se deberían contabilizar seis horas lectivas en los centros de tres o menos líneas de ESO y nueve horas lectivas en los de cuatro o más líneas de ESO. ¿Por qué se incrementan las horas y se describen en los mismos términos las funciones de estos profesionales que en las normativas anteriores? ¿Qué sucedió para que la Administración accediera a ese aumento de horas no lectivas dedicadas a la coordinación y la colaboración? Sin duda, la constatación que con tres horas semanales era materialmente imposible ejercer el tipo de coordinación y colaboración que se pedía al psicopedagogo de un centro.

Pero el movimiento basculó de nuevo hacia atrás en la normativa del curso 1999-2000. En ella ya no se menciona el tema de la coordinación en las tareas de tutoría, ni tampoco, de forma explícita, la colaboración con los departamentos, EAPS, etc. Ahora todavía aumenta más la imprecisión puesto que el psicopedagogo será "el responsable de coordinar las actividades docentes que el centro establezca para la atención del alumnado con necesidades educativas especiales" "10. ¿Y cuáles serán las que el centro establezca? ¿Es el equipo directivo y sus decisiones "el centro establecedor?

Respecto a la "colaboración", ahora se dice que se ha de producir con los diversos órganos que trabajan en la atención a la diversidad de los alumnos con necesidades educativas especiales de acuerdo con las previsiones establecidas en el PCC (Proyecto Curricular de Centro). No se mencionan departamentos, ni EAPs, pero se sobreentiende que con éstos ha de continuar la colaboración, pero también con la comisión de atención a la diversidad, con el coordinador pedagógico, con los equipos docentes y con los tutores, etc. Este aparente incremento de órganos con los que colaborar y de actividades a coordinar se traduce, sin embargo, en las mismas horas de reducción (seis y nueve horas dependiendo de la dimensión del centro) pero

10. La cursiva es nuestra. Normativa del curso 1999-2000. Ibídem, apartado 5.4.1. 
añadiendo que esas horas se han de asignar "a las funciones de atención individualizada a alumnos y de coordinación de este profesorado" ${ }^{11}$. Si la atención individualizada se definía antes - en la función " $a$ - como la docencia en pequeño grupo de alumnos con necesidades educativas especiales, y también la preparación de materiales y actividades adaptadas en colaboración con el resto del profesorado, entonces ¿todas las horas descritas se emplearán en esa función no quedando, pues, horas para el resto de funciones de coordinación y de colaboración previstas? ¿O esas horas deberán "repartirse" entre las dos o tres funciones? Si así se interpreta, el paso atrás de esa normativa de curso ${ }^{12}$ en comparación con las normativas anteriores resulta evidente. Por eso, si añadimos aquí el mayor peso de las funciones de docencia en los créditos comunes y el menor en los créditos variables, excepto en los de refuerzo que ahora son prioritarios y, también, la falta de concreción de las funciones de coordinación y colaboración - y su, en la práctica, falta de horario preestablecido-, no resulta difícil imaginar cuál es la actual situación de los psicopedagogos en los centros. Tanto desde el punto de vista de la fijación de sus funciones profesionales como de las condiciones de trabajo (horarios, reconocimiento específico de su especialización, no reconocimiento de sus potencialidades como asesor y dinamizador pedagógico del centro, como experto en diseño curricular y en innovación educativa, etc.) la evolución de las distintas normativas de curso" indican la clara tendencia a no reconocer un perfil profesional propio al psicopedagogo de Secundaria.

Hemos de mencionar aquí otro detalle interesante de la evolución de la normativa. En las anteriores a la del curso 1999-2000 se decía que el psicopedagogo impartía la docencia "preferentemente" en la etapa de la Educación Secundaria Obligatoria. Pero eso no impidió a buena parte de los psicopedagogos de los centros impartir la asignatura de Psicología del nuevo Bachillerato y también algunos créditos relacionados con esta materia en esa etapa educativa. Parecía tener toda la lógica del mundo que si había un profesional indicado en los centros de secundaria para impartir esa materia ése era el de la especialidad de Psicología y Pedagogía. Pues bien, en la normativa del curso 1999-2000, y por extensión en la del curso 2000-2001, se dice que este profesorado «imparte docencia en la etapa de la Educación Secundaria Obligatoria" ${ }^{13}$. Y nada más. Ha desaparecido el "preferentemente». Los directores de los centros ya pueden, desde entonces, decidir libremente que el psicopedagogo sólo es docente de la Eso.

11. Ibídem, apartado 5.4.1.

12. Recordemos que es la vigente también en el curso 2000-2001 puesto que no ha sufrido modificacions en relación con la del año anterior.

13. Ibídem, apartado 5.4.1. 


\section{4. ¿MÁs CONCRECIÓN NORMATIVA O MÁS AUTONOMÍA DE CENTRO?}

Después de ver la poco afortunada evolución de la normativa reguladora de las funciones profesionales de los psicopedagogos en los IEs de Cataluña, nos hemos de preguntar el porqué de esta situación y, a continuación, qué funciones y marco de actuación interesaría más potenciar a partir de ahora desde el punto de vista de mejorar la aportación pedagógica de estos profesionales en los centros. Pensamos así mismo que nuestras reflexiones sobrepasan el marco de una Administración educativa concreta - en este caso Cataluña-y pretendemos que sirvan para incitar la reflexión del papel que deben jugar los psicopedagogos en todos los centros de Secundaria estatales.

Empecemos por una primera constatación: la evolución errática y con algunos cambios desconcertantes, casi de año en año, de una normativa que, sobre el papel, debería pretender fijar las funciones profesionales de un colectivo que se estrena en un campo profesional propio, no nos debería sorprender demasiado. Estudiando el diverso grado de influencia y de presión de algunos lobbys bien determinados actuando cerca de la Administración en momentos cruciales, y teniendo en cuenta también de manera general el clima creado en el sector de la Secundaria Obligatoria por la aplicación real y extensiva a todos los centros de la reforma, se explicarían muchos de esos cambios aparentemente desconcertantes indicados en el apartado anterior. Pero ello nos llevaría a un análisis que se escapa de las posibilidades de este artículo. Sin embargo, podemos afirmar que la evolución profesional de los psicopedagogos en los centros de Secundaria ha estado, está y estará en un futuro próximo muy condicionado por la propia evolución, contradicciones y aciertos — que también los consigue- de la reforma educativa y, más en concreto, de la ESO como etapa a consolidar; de la evolución de la ESO y de todo el sistema educativo en general. Y dependerá de ámbitos como la atención a la diversidad, el marco de convivencia con los alumnos adolescentes, del modelo de orientación educativa y de intervención psicopedagógica que al final se imponga (Del Rincón, 1998: 133), del papel que pueden desarrollar en los centros las nuevas tecnologías de la información, etc. Todo ello va a influir de forma determinante, pero el trabajo de futuro dependerá finalmente de que los propios psicopedagogos sean capaces de analizar y estudiar detenidamente la realidad sociocultural de su centro (Sánchez Palomino, 1999: 188). Por tanto, dependerá también de la composición sociocultural de su alumnado, y de que ellos, los psicopedagogos, tengan una formación (inicial y permanente) mucho mejor que la de ahora y más adaptada a los retos profesionales concretos pero "sin olvidar que una excesiva especialización puede constituir un inconveniente en el encuentro de salidas profesionales, cuando el mercado profesional resulta "opaco" para los pedagogos" (Sarramona, 2000a: 107) - y también para los psicopedagogos, donde la docencia en la Secundaria no deja de ser por el momento una salida reservada a muy pocos 
profesionales y con fundadas dudas sobre su expansión futura ${ }^{14}$-; y, finalmente, que su actividad se oriente claramente a la acción educativa pero con espíritu investigador puesto que, "en educación, investigar es actuar" (Fernández Sierra y Carrión, 1999: 78) y si alguien necesita vincular más su práctica profesional a la investigación educativa para poder orientar la práctica éste es, sin duda, el psicopedagogo de un centro.

Cuando la figura del psicopedagogo se incorporó a los centros de Secundaria levantó una serie de expectativas que en buena parte se han .visto defraudadas. Algunos estudios indican que los equipos directivos de los institutos cualifican entre poca y nula la colaboración que reciben de los psicopedagogos en aspectos como la planificación y la organización de los centros, en especial, las referidas a la acción tutorial y a la evaluación (Martínez i No, 2000: 46). Eso no debería extrañarnos dadas las funciones prioritarias que la Administración ha otorgado hasta este momento a estos profesionales. Al mismo tiempo no podemos olvidar que «ni los docentes con más antigüedad ni el equipo directivo, suelen mostrarse propensos a modificar ciertas normas acrisoladas" (Angulo, 1995: 447 citando a Rosenholtz). La sensación general entre los profesores de Secundaria — con todas las excepciones que se quiera - sigue siendo que los psicopedagogos, si son especialistas de algo en estos centros, lo son en ocuparse de los alumnos con necesidades educativas especiales y, por extensión - y gracias a una patética generalización-, sobre el conjunto de alumnos que resultan "problemáticos" o "Conflictivos" en los actuales centros de Secundaria. Y aquí se ponen en un mismo cajón los alumnos con deficiencias psíquicas o físicas y todos los otros que presentan un grado u otro de retraso o fracaso escolar. Y, naturalmente, también se incluyen todos los otros "que no estudian", "que no bacen nada", "que molestan", "que han lanzado la toalla", "que resultan inaguantables e insolentes", "que son malos (si, si, de maldad)", etc. Es decir, todos aquellos alumnos que, según un amplio sector del profesorado — sobre todo el que proviene del antiguo Bup y COU-, no deberían estar en los institutos puesto que éstos no deberían convertirse en centros de acogida social o de prevención o contención de la predelincuencia" de muchos de los chicos y chicas que asisten, "por fuerza", a los actuales institutos hasta los dieciséis años. El psicopedagogo, el "especialista" en esos problemas, debería saber qué hacer con estos chicos. ¿No viene, su figura profesional, muy vinculada con la nueva reforma? Pues bien — piensa ese colectivo de profesores-, que sea él quién lo resuelva, o al menos que se ponga delante y actúe; que no teorice y que no asesore;

14. En Cataluña no suele haber más de uno por centro y todavía si el centro lo reclama. Está por ver si la intención de la Administración es dotar de más psicopedagogos a los centros potenciando la labor de asesoramiento, intervención y orientación educativa o bien cortar o retardar el proceso. En la convocatoria de oposiciones para la provisión de plazas de funcionarios docentes de la Educación Secundaria de marzo de 2001 no hay ninguna plaza prevista de psicopedagogo, lo que constituye sin duda una pésima noticia desde la perspectiva que impulsamos en este trabajo y que mucho nos tememos signifique la segunda opción de las señaladas más arriba. 
que no sólo colabore o coordine sino que ejerza docencia directa y cuanta más — más alumnos a la vez- mejor. Y que la haga a la ESO, naturalmente, puesto que al Bachillerato no se le necesita para nada. En el Bachillerato - siguen pensando estos profesores - ni teníamos ni tenemos los problemas que la "reforma" nos ha traído con esto de atender toda la diversidad de alumnos, por lo tanto, no necesitamos psicopedagogos.

Con esta actitud de fondo no son de extrañar los cambios en la normativa. Ė colectivo de profesores de Lengua, Matemáticas, Ciencias Naturales, Ciencias Sociales, etc., es mucho más numeroso y, por tanto, con mucho más poder de influencia que un colectivo de algunos centenares de psicopedagogos que, en muchos institutos, actúan en solitario y que todavía, no sólo no han conseguido crear un departamento de orientación en sus centros, sino ni tan sólo están inscritos en alguno. Según un estudio de la Inspección de Educación sobre una muestra de centros de toda Cataluña en el curso 1994-1995, el 23\% de los psicopedagogos no estaban inscritos a ningún departamento, el 62\% estaba inscrito en el de Lenguas y el 15\% restante en el de Ciencias Sociales (Martínez i No, 2000: 30). En los últimos años, afortunadamente, esta situación va cambiando y ahora los departamentos de orientación comienzan a estar presentes en los centros al "margen" de la normativa $^{15}$. No obstante, los cambios se generaron independientemente de la normativa puesto que ésta recoge muy a menudo previsiones de tipo teórico y otra cosa distinta son las dinámicas de la realidad del cada día de los centros. Y esta realidad nos está indicando claramente la conveniencia de contar en los institutos con departamentos de orientación donde ubicar, con personalidad propia, tanto los psicopedagogos como los otros profesionales que realizan tareas afines (maestros de Pedagogía Terapéutica, profesores de vac, del servicio de compensatoria, etc.).

Los departamentos de orientación son necesarios en los institutos porque los departamentos y los seminarios didácticos, lejos de haber cambiado su lógica de funcionamiento y organización interna - muy vertical, con la disciplina como punto de encuentro de profesionales y no con el grupo clase como centro de las reuniones y las dinámicas pedagógicas - han continuado ostentando el máximo poder de decisión en los institutos. Estos departamentos, constituidos como auténticos reinos de taifas, son también el refugio de pequeños privilegios y egoísmos particulares. En consecuencia, no debe extrañar que el psicopedagogo - que representa siempre en los centros la preeminencia de la perspectiva pedagógica por delante de la que tienen la disciplina académica como eje rector de actuacióntenga dificultades para relacionarse con los departamentos y que el diálogo con ellos sea prácticamente inexistente (Martínez i No, 2000: 112). Todavía son muchas

15. En Cataluña no se optó por un modelo de creación de departamentos de orientación dentro de los institutos puesto que se creyó que esa función, la de orientación, la habían de desarrollar, preferentemente, los profesionales del EAP asesorando a los tutores en los centros y no mediante los psicopedagogos. 
las resistencias en los centros de Secundaria a otorgar un mayor protagonismo a la organización horizontal que sería la más idónea, desde el punto de vista pedagógico, para tomar las decisiones colegiadas sobre los diversos asuntos que inciden en la enseñanza en una etapa que es obligatoria y básica para toda la población como representa la ESO. Modificar esta situación de hecho consume multitud de energías que se gastan en múltiples batallas internas entre los profesores. Esas batallas "queman" mucho más que la relación conflictiva con el alumnado. De hecho, la relación con los colegas docentes resulta ser una variable muy determinante en el mencionado malestar docente (Esteve, Vera y Sacristán, 1988: 95, donde citan a muchos otros autores).

La actual imagen profesional de los psicopedagogos en los centros, ¿es debida a la inconcreción, ambigüedad, constantes cambios y contradicciones de las normativas que han regulado las funciones de estos profesionales? O bien, como justifica la Administración ("no bemos fijado mucho las funciones y las bemos dejado abiertas para que cada centro pueda disponer del psicopedagogo para efectuar aquellas actividades y funciones que puedan optimizar mejor su preparación y capacidad profesional " $)^{16}$, ¿sería peor si se hubieran delimitado mucho más las funciones y los horarios desde la normativa administrativa? Es difícil responder a esa pregunta puesto que aquello que no se ha llevado a la práctica resulta imposible de valorar. Sin embargo, mucho nos tememos que unas funciones más cerradas y concretas desde la Administración tampoco habrían dejado contento a nadie y sí habrían establecido un precedente de tipo administrativo que, en comunidades tan burocratizadas como las nuestras, resultaría luego muy difícil de cambiar. Pero si, por el contrario, se opta por convicción pedagógica por dejar parcialmente abiertas esas funciones se han de asumir todas las consecuencias. No sólo a medias. Es decir, lo que no tiene justificación es ir cambiando matices, introduciendo u omitiendo sectores del discurso normativo a golpe de la última influencia con más poder ejercida sobre la Administración. Eso no responde a ninguna política educativa definida. Por eso dudamos que el Departamento de Enseñanza en Cataluña tenga un modelo muy determinado del papel profesional que deberían ejercer los psicopedagogos en los centros. Quizás era lógico que no lo tuviera durante los primeros años, puesto que ni los propios psicopedagogos tenían claro cuáles deberían ser sus prioridades funcionales y profesionales en el ejercicio de, precisamente, una profesión nueva en un marco educativo también innovador. Pero resulta mucho más preocupante que, después de seis o siete años de existencia de los psicopedagogos en los centros de Secundaria, la Administración titubee, no concrete, cuando no se contradiga, en referencia a sus competencias profesionales. El psicopedagogo, por más profesor que sea de secundaria, no debe ser definido principalmente como "un profesor más del centro". Sencillamente porque

16. En unos términos aproximados a éstos se expresó un técnico del Departamento de Enseñanza en un cursillo impartido a los psicopedagogos de Secundaria durante el curso 1996-1997. 
nadie cree que lo sea, ni él mismo. Su docencia prioritaria no ha de ser el refuerzo de Matemáticas y Lengua. No se pueden echar por la borda las potencialidades de asesoramiento, intervención y orientación (psicopedagógica, didáctica, curricular, organizativa, personal, académica, profesional, de tutoría, etc.) que, teóricamente al menos, contiene su formación académica y, debería tener también, su experiencia profesional.

Pero todavía no hemos contestado a la pregunta del presente apartado. Tal como están ahora las cosas, ¿resultaría más pertinente pedir más concreción normativa o, por el contrario, deberíamos aprovecharnos de la ambigüedad existente y aún sería conveniente reclamar más desregulación normativa para poseer, como psicopedagogos, una mayor polivalencia y mejor adaptación en la respuesta profesional dentro de cada contexto singular de centro? Somos partidarios, después de analizar los pros y contras, que las funciones de los psicopedagogos se definan en el marco del PCC (Proyecto Curricular de Centro) de cada instituto pero siguiendo ya unas directrices que habría establecido el PEC (Proyecto Educativo de Centro) dentro de los apartados correspondientes a la definición de los principios organizativos y de gestión del instituto y el de los principios pedagógicos que el centro da prioridad adecuándolos a su propia realidad ${ }^{17}$. La Administración debería dar libertad de fijar el horario de los psicopedagogos y sus funciones en el centro a los equipos directivos para que, junto con la opinión del EAP de zona, la supervisión de la Inspección y, evidentemente, la opinión del psicopedagogo y del propio departamento de orientación del centro, se elaborara una propuesta que fuera aprobada también por la Comisión de Atención a la Diversidad del centro. Ese horario y esas funciones serían evaluadas periódicamente a partir de los objetivos fijados en el seno de la propia comisión y de acuerdo con el equipo directivo. Otra cosa sería replantear los integrantes de esta comisión donde nosotros creemos más adecuado integrar al coordinador de la ESO o a los coordinadores de ciclo - en el caso de la existencia de un coordinador por ciclo- que no a los jefes de los departamentos de las áreas instrumentales, puesto que pensamos que esta propuesta resulta más lógica desde el punto de vista pedagógico y después de haber defendido ya en otro lugar (Roca, 1999b: 138) una organización prioritaria de la estructura horizontal en la etapa de la Eso (equipos docentes de curso, equipos de tutores, equipos de ciclo) y una disminución del poder de influencia de la estructura vertical (departamentos y seminarios didácticos). Creemos que, en esa etapa, no debería ser la lógica de la materia a enseñar la que prevaleciera sobre la de las necesidades educativas de cada alumno y de cada grupo de alumnos.

En Cataluña la Administración educativa está poniendo en marcha, dentro de lo que se conoce como el programa "Educació 2000-2004", la promoción e implantación

17. Decreto 199/1996, de 12 de junio, de aprobación del Reglamento orgánico de los centros docentes públicos que imparten Educación Secundaria y Formación Profesional de grado superior. Artículo 8.3., apartados b y $c$. 
de planes estratégicos dentro los cuales cada centro pueda adoptar las medidas singulares que mejor le convengan para conseguir los objetivos de su plan. De momento, durante el curso 2000-2001, esa promoción se ha restringido a un número reducido de institutos, pero la idea es que en el marco de la evaluación interna de los centros, acabada la implantación generalizada de la etapa de la Educación Secundaria Obligatoria en el curso 1999-2000, cada centro, en el marco de este proceso de reflexión y evaluación interna, pueda elaborar su propio plan estratégico del período 2000-2004. Esos planes han de tener como principal finalidad el aumento de la calidad educativa global del centro y, muy especialmente, conseguir que el alumnado adquiera las competencias básicas de las etapas obligatorias (Sarramona, 2000b). El plan estratégico ha de definir unos objetivos a partir de la realidad del centro y podrá "incidir en la organización y adaptación del currículum, en el perfil y sistema de provisión específica de determinados puestos de trabajo, en el reglamento de funcionamiento del centro y en las características de los recursos materiales" (Departament d'Ensenyament, 2001: 1). En esta perspectiva, si cada centro puede incidir sobre el perfil de determinados puestos profesionales para que se adecuen a su plan estratégico, no vemos ningún inconveniente que al psicopedagogo del instituto (y el maestro de Pedagogía terapéutica si lo hay) se le exija un perfil profesional lo más adaptado posible a las prioridades y opciones que se defiendan en el plan estratégico del centro. Además, si el propio psicopedagogo ha sido un elemento activo en la elaboración de ese plan sus funciones y perfiles profesionales se habrán definido, por una parte atendiendo a las necesidades, objetivos y previsiones realizadas en el plan estratégico y, por otra, sus funciones específicas podrán acomodarse a su propio perfil profesional al margen de las normativas administrativas anteriores, es decir, que ahora podrán estar en conjunción con su auténtica preparación profesional y especialización académica.

Vemos, pues, en la puesta en marcha de estos planes estratégicos, una magnífica oportunidad para librarnos de normativas que limitan acciones, que resultan contradictorias desde el punto de vista pedagógico y que ayudan a crear un cierto desconcierto y una determinada mala fama de la Pedagogía. Para algunos, la Pedagogía académica estaría ejerciendo un excesivo poder de influencia en la escuela imponiendo discursos teóricos por encima de la experiencia cotidiana del profesor (Cardús, 2000: 48-49). Resulta significativo que un sociólogo de la categoría de Cardús, siempre atento a captar agudamente la opinión social, exprese esa sensación. Quizás nos deberíamos preguntar qué hemos hecho entre todos para ganar esta fama. Es más, muchos han visto en los psicopedagogos - ¿será por el nombre?a los auténticos portavoces de esa influencia y de ese supuesto poder que, en caso de existir en algún grado, sería sin duda mucho más teórico que práctico. Y no afirmo que no tengamos - los psicopedagogos de los centros y los psicopedagogos, psicólogos escolares y pedagogos de universidad - una buena parte de responsabilidad en haber contribuido a crear ese prejuicio social. La distancia entre los problemas prácticos y cotidianos de los profesores en sus centros respecto a los discursos conceptualistas, y muy alejados de la batalla del día a día de nuestras aulas, de muchos profesores universitarios que viven en una especie de nube de algodón 
viajando por encima de realidades que sólo conocen desde sus visiones aéreas y no de a pie, esa distancia decimos, es bien real y contrastable. Todo ello ha contribuido, sin duda, a este desprestigio.

Pero reconocido esto, la penetración de la Pedagogía en la formación permanente del profesorado de Secundaria no produce precisamente excesos didactistas y reformadores. No los puede producir sencillamente porque su poder de influencia es mínimo a la luz de lo poco interesados que se muestran la mayoría de profesores del antiguo sistema educativo para actualizar sus conocimientos pedagógicos - que ya eran realmente deficitarios en su formación inicial-y que ahora, con la necesidad imperiosa de buscar cambios e innovaciones metodológicas y didácticas - y en el fondo de concepción pedagógica, de mentalidad profesionaldelante los problemas que tenemos planteados en las aulas de la Eso, ahora, no muestran precisamente ningún entusiasmo por el reciclaje o la actualización pedagógica. Y el psicopedagogo no está al margen de esta cultura antipedagógica imperante, al contrario, la sufre en sus propias carnes y muy frecuentemente se encuentra en el medio, entre una reforma que no ha conseguido implicar a los profesores

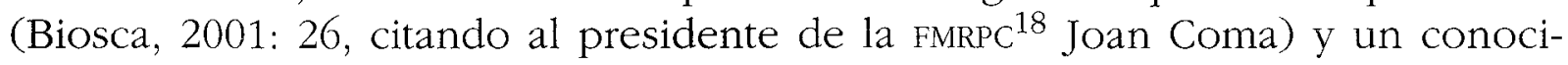
miento psicopedagógico que es percibido, por esos mismos profesores, como un discurso teórico, poco práctico, idealista, academicista o ingenuo delante de un conocimiento - el suyo- que dicen que se muestra más realista al nacer, sobre todo, de la experiencia y no de la teoría. Eso sí, desde esa pretendida "experiencia" no se para de reclamar la exclusión del sistema de los alumnos que no siguen, que no hacen nada porque no quieren o no saben estudiar, etc. ¿En qué se muestra eficaz pues esa "acumulación de experiencia" delante de estos auténticos retos educativos y profesionales?

En definitiva, acogiéndonos a la letra que propone la filosofía del plan estratégico de centro pensamos que los psicopedagogos han de tener un papel de primer orden en la reclamación de que su centro comience cuanto antes dicho plan. $\mathrm{Y}$ apostamos porque se implique activamente en todos sus apartados y en, sobre todo, la definición ajustada de su perfil profesional a las características del centro - en especial del análisis de las mayores necesidades educativas de su alumnadoy a las funciones y objetivos que él mismo, como profesional y a partir de su formación y experiencia, pueda asumir dentro de ese plan. Representa, pues, una excelente ocasión para que, poco a poco, se pueda ir creando un perfil profesional de psicopedagogo más adaptado a sus posibilidades reales de intervención en los centros. Que también pasará por la docencia, pero que, sobre todo, se basará, a nuestro entender, en la preeminencia de las funciones de asesoramiento, evaluación psicopedagógica y orientación personal, académica y profesional al alumnado y, también, de la orientación pedagógica, curricular e innovadora a los profesores del centro.

18. Federación de Movimientos de Renovación Pedagógica de Cataluña. 
5. Algunas propuestas de futuro CON RELACión al perfil profesional y a las FUNCIONES DE LOS PSICOPEDAGOGOS EN LOS IES

Ya hemos mencionado que, en Cataluña, existe ahora una buena ocasión para superar esa primera etapa profesional que ha servido para introducir el profesional de la psicopedagogía en los centros de Secundaria. Aún es muy pronto para afirmar que la figura profesional se ha consolidado a pesar de los frecuentes vaivenes normativos. Pero ahora se acercan momentos decisivos donde los centros deberán revisar sus documentos y sus objetivos elaborando planes de actuación concretos y singulares. Ya hemos defendido antes que los psicopedagogos intervengan activamente para dar contenido y forma pedagógica de amplio calado a estos proyectos. Es ahora cuando tendrá la ocasión de demostrar que es en el asesoramiento, en la implicación práctica y teórica de los proyectos de actuación pedagógica, ofreciendo ideas y aportando innovaciones metodológicas y de enfoque educativo, sobre todo, vinculados a las nuevas tecnologías de la información, que será en todas estas actividades y tareas donde deberá demostrar que su papel profesional en el centro se optimiza precisamente en estos quehaceres sin olvidar el contacto, la evaluación y la implicación también docente con los alumnos. No estamos planteando esto para proclamar que los psicopedagogos se han de liberar de la docencia de las materias instrumentales y han de pasar a ejercer de técnicos o expertos teóricos que asesoran a quien no sabe. Nada de esto. Se trata de que todos descubran - y el psicopedagogo en primer lugar, que se autoconvenza- que sus potencialidades profesionales se han de ejercer en multitud de registros y que ha de demostrar una enorme polivalencia profesional, pero también una gran flexibilidad y agilidad profesional para incorporar rápidamente aquellos avances tecnológicos y didácticos que han de poder facilitar la labor de enseñanza de los profesores y la del aprendizaje de los alumnos.

De forma muy esquematizada proponemos posibles funciones profesionales que ahora no recoge la normativa administrativa oficial, pero que nosotros consideramos interesante tenerlas en cuenta en una nueva fase de definición del perfil profesional, Un perfil contextualizado a cada centro y a sus concretas necesidades educativas:

a) En colaboración con los profesionales del EAP, realizar —en todas sus fases- evaluaciones psicopedagógicas a los alumnos con necesidades educativas especiales.

b) Asesorar y complementar con el tutor del grupo de alumnos la orientación personal, académica y profesional (o preprofesional para ser más exactos) del alumnado.

c) Coordinar, impulsar, orientar y supervisar la política educativa del centro en relación con la atención a la diversidad. Esto supondría también, como mínimo, codirigir (con el coordinador pedagógico) la Comisión de Atención a la Diversidad del instituto.

d) Asesorar y participar de forma prioritaria en el diseño curricular del centro en todos y cada uno de los elementos que lo integran. Eso supone ser 
un miembro activo y clave de la comisión de seguimiento y actualización continua del Pcc. Este Proyecto Curricular de Centro se ha de concebir no como un documento sino como aquel instrumento didáctico que nos permita, entre otras funciones, conocer, contrastar, observar y valorar el trabajo docente (Roca, 1997: 146).

e) Asesorar y colaborar en la atención a la diversidad cultural del alumnado asesorando y colaborando con el profesorado en la acogida y la integración de la población inmigrada (Montané, 2000: 11).

f) Implicarse en el diseño, el seguimiento y la evaluación de la normativa de convivencia del centro (problemas de comportamiento y disciplina; derechos y deberes de alumnos y profesores, medidas correctivas pedagógicas y no sancionadoras, etc.).

g) Apoyar, asesorar y fomentar la formación permanente de todos los docentes del centro siendo un motor impulsor de la investigación y la innovación escolar y educativa en su centro (Angulo, 1995: 427).

h) Colaborar en la elaboración, la adaptación y la evaluación de materiales, recursos didácticos y metodológicos.

i) Implicarse activamente en la elaboración del plan estratégico del centro donde, entre otros muchos temas en que puede colaborar, deberá ayudar a rediseñar sus funciones profesionales dentro del instituto con el objetivo de optimizarlas a partir de su propia formación y experiencia y de las necesidades concretas del centro.

Esas funciones sólo representan un ejemplo de aquellas tareas que un psicopedagogo de Secundaria podría realizar al margen de un seguidismo acrítico de la normativa y más en concordancia con un proyecto de centro específico (como puede ser el plan estratégico de centro antes mencionado). En cualquier caso el camino ni es ni será fácil. El campo profesional todavía está por ganar y consolidar. Y sólo se avanzará significativamente en la medida que los psicopedagogos sean percibidos por todos los componentes de la comunidad educativa como claros contribuyentes a la mejora de la calidad educativa del centro a partir de sus aportaciones y asesoramientos profesionales, pero también de su capacidad para trabajar en equipo y, sobre todo, para establecer puentes entre las distintas culturas profesionales coexistentes en un centro de Secundaria. La tarea, por costosa e incierta, resulta apasionante y requiere unas enormes dosis de entusiasmo, de capacidad de formación y evaluación docente y no docente constante como, por otra parte, lo requiere cualquier otro profesor (Roca, 1999a). Pero, más que nada, exige partir del convencimiento de que todo esfuerzo por mejorar la educación de nuestros jóvenes, y de entre ellos los más desfavorecidos, tiene su compensación a medio o largo plazo. Compensación para ellos que dispondrán de más recursos personales para afrontar los nuevos cambios sociales y compensación para nosotros que observaremos que nuestro esfuerzo pedagógico se ha traducido en un bien social, cultural y, por encima de todo, personal. 


\section{BIBLIOGRAFÍA}

Angulo, J. F. (1995): "El papel innovador de los/las psicopedagogos/as en los centros de secundaria obligatorian. En Fernández Sierra, J. (coord.): El trabajo docente y psicopedagógico en educación secundaria. Ed. Aljibe, Málaga, pp. 427-458.

BiosCA, C. (2001): "Mestres progressistes admeten que no s'ha aconseguit implicar els docents en la LoGse". Avui. Dijous, 18/01/01, 26.

CARdús, S. (2000): El desconcert de l'educació. $3^{a}$ edició. Ed. La Campana, Barcelona (próxima edición castellana en Ediciones B).

DEPARTAMENT D'ENSENYAMENT (2001): Organització i funcionament de centres docents. Curs 2000-2001. Departament d'Ensenyament, Generalitat de Catalunya, Barcelona.

ESTEVe, J. M.; Vera, J. y SACRISTÁN, D. (1988): "La comunicación entre los profesores: un difícil laberinto y una vía de autorrealización”. En Sarramona, J. (ed.): Comunicación y educación. Ed. CEAC, Barcelona, pp. 81-102.

Fernández SierRa, J. y Carrión, J. J. (1999): “De orientadores a psicopedagogos: la formación de un nuevo profesional.. En Fernández Sierra, J. (coord.): Acción psicopedagógica en Educación Secundaria: Reorientando la orientación. Ed. Aljibe, Málaga, pp. 65-78.

MarTínez i No (2000): Perfil professional i funcions dels/les especialistes de Psicologia i Pedagogia dels instituts d'ensenyament secundari: anàlisi de la situació actual, disseny d'una pauta de supervició i propostes de futur. Treball de Recerca. Mestratge en Supervisió i Avaluació de l'Educació, Facultat de Ciències de l'Educació, Universitat Autònoma de Barcelona (documento inédito).

Montané, J. (coord.) (2000): Perfil i funcions del psicopedagog. Facultat de Ciències de l'Educació, Universitat Autònoma de Barcelona.

Rincón, B. del (1998): El psicopedagog en el centre de secundària. Suggeriments i propostes. Ed. Estel, Barcelona.

ROCA, E. (1997): El desarrollo curricular en secundaria. Ed. CEAC, Barcelona.

- (1999a): "Hacia una cultura de la evaluación docente en secundaria. Propuestas de evaluación de la práctica docente y de la formación inicial y permanente del profesorado". Comunicaciones. VII Congreso Nacional de Teoría de la Educación. Cambio educativo: presente y futuro. Servicio de Publicaciones de la Universidad de Oviedo, pp. 78-88.

- (1999b): "L'avaluació de l'aprenentatge: algunes reflexions des de la pràctica”. En VArios Autores: Avaluació i educació. Documents 8. Consell Escolar de Catalunya, Generalitat de Catalunya, Barcelona, pp. 126-145.

SÁnchez Palomino, A. (1999): “El psicopedagogo y la atención a las necesidades educativas especiales en secundaria. En Fernández Sierra, J. (coord.): Acción psicopedagógica en Educación Secundaria: Reorientando la orientación. Ed. Aljibe, Málaga, pp. 171-198.

Sarramona, J. (2000a): Teoría de la Educación. Reflexión y normativa pedagógica. Ed. Ariel, Barcelona.

- (2000b): "Competencias básicas al término de la escolaridad obligatoria". Revista de Educación, 322, 255-288. 